REGARDS

SUR L'ECONOMIE ALLEMANDE

BULLETIN ECONOMIQUE DU CRAC

\section{Regards sur l'économie allemande}

Bulletin économique du CIRAC

$69 \mid 2004$

Varia

\title{
Financement et vieillissement : le Mittelstand en mutation
}

Markus Gabel

\section{OpenEdition}

Journals

Édition électronique

URL : http://journals.openedition.org/rea/3313

DOI : 10.4000/rea.3313

ISBN : 978-2-8218-0834-8

ISSN : 1965-0787

Éditeur

CIRAC

Édition imprimée

Date de publication : 1 décembre 2004

ISSN : 1156-8992

\section{Référence électronique}

Markus Gabel, « Financement et vieillissement : le Mittelstand en mutation », Regards sur l'économie

allemande [En ligne], 69 | décembre 2004, mis en ligne le 27 janvier 2009, consulté le 30 avril 2019

URL : http://journals.openedition.org/rea/3313 ; DOI : 10.4000/rea.3313 


\title{
Financement et vieillissement : le Mittelstand en mutation
}

\author{
Markus Gabel
}

Plus que les grandes entreprises, les PME allemandes ont souffert de l'atonie de la croissance, de la faiblesse de la consommation et des difficultés financières du secteur bancaire. Le Mittelstand est également marqué par l'incertitude en matière de transmission d'entreprises, particulièrement forte dans un pays où de nombreuses PME sont dorénavant contraintes de trouver un successeur en dehors du cercle familial. S'y ajoute l'épineuse question du financement. Le nouvel accord sur les capitaux propres des banques, Bâle II, remodèle la relation banque-entreprise, si centrale outre-Rhin, et modifie profondément les conditions de financement d'un Mittelstand qui reste encore trop souvent handicapé par sa traditionnelle faiblesse de fonds propres. Si le fameux credit crunch n'a finalement pas eu lieu, les banques sont devenues plus sélectives dans le choix de leurs crédits et obligent les PME à s'ouvrir à des schémas de financement plus innovants - une voie également empruntée par les banques via la True-sales-Initiative qui vient de démarrer...

La notion du Mittelstand est spécifique à l'Allemagne. Si ailleurs on parle simplement de PME et TPE, outre-Rhin, le Mittelstand mêle également des aspects sociologiques et psychologiques. La définition qu'en donnait Ludwig Erhard reste d'actualité : "Le Mittelstand ne peut guère être mesuré en seuls termes matériels, car il est davantage empreint d'un état d'esprit et d'une attitude spécifique qui s'exprime dans le processus socio-politique ». Les entreprises de taille moyenne se trouvent dans toutes les branches, y compris les professions libérales, et bénéficient d'un lobbying politique considérable : partis politiques, fédérations professionnelles, etc. ont une division dédiée (ainsi la Mittelstandsvereinigung de la CDU). Le Mittelständler typique réunit propriété et responsabilité, gère le risque et est indépendant. II est organisé sous forme d'entreprises en nom personnel ou sous loi civile (Gesellschaft des bürgerlichen Rechts) ; seulement $15 \%$ de ces PME sont des $\mathrm{GmbH}$ (Sarl), les SA étant encore plus rares.

Mittelstand : des critères culturels plus que de taille

\begin{tabular}{|c|c|c|c|}
\hline \multicolumn{4}{|c|}{ Les définitions du Mittelstand : un enjeu non négligeable } \\
\hline \multicolumn{4}{|c|}{ Définition de I'Institut du Mittelstand et de la KfW (environ 3,3 millions d'entreprises) } \\
\hline Taille de l'entreprise & Salariés & Chiffre d'affaires & Répartition \\
\hline Petite & jusqu'à 9 & jusqu'à 1 million $€$ & $84,8 \%$ \\
\hline Moyenne & 10 à 499 & 1 à 50 millions $€$ & $14,8 \%$ \\
\hline Grande & 500 et plus & 50 millions $€$ et plus & $0,4 \%$ \\
\hline \multicolumn{4}{|c|}{ Autres définitions } \\
\hline \multicolumn{4}{|c|}{$\begin{array}{l}\text { En } 2001 \text {, la Commission européenne a changé le règlement } 96 / 280 / C E \text { et conseille, à partir du } 1^{\mathrm{er}} \text { janvier } 2005 \text {, l'application de la } \\
\text { définition suivante : un effectif de } 10 \text { à } 249 \text { salariés, un CA de } 10 \text { à } 50 \text { millions } € \text { ou un bilan entre } 10 \text { et } 43 \text { millions } € \text {. S'y ajoute le } \\
\text { critère de "l'indépendance " : moins de } 25 \% \text { de fonds propres ou de droits de vote contrôlés par une entreprise tierce. L'Institut } \\
\text { pour le Mittelstand (IfM) estime que } 95 \% \text { des PME allemandes entrent dans cette catégorie. } \\
\text { L'application de ce conseil réduit sensiblement la taille du Mittelstand en Allemagne : sa contribution à la création de valeur et à } \\
\text { l'emploi serait ainsi modifiée, ce qui pourrait avoir à l'avenir des conséquences quant à la représentation politique du secteur et, } \\
\text { éventuellement, favoriser les très grandes entreprises industrielles. }\end{array}$} \\
\hline \multicolumn{4}{|c|}{$\begin{array}{l}\text { D'autres définitions existent, par exemple pour la comptabilité (voir le Journal officiel de la CE n L } 283 \text { du } 27 \text { octobre } 2001 \text { ) et } \\
\text { selon les traditions nationales. Ainsi, en France, est considérée comme une PME toute entreprise de moins de } 499 \text { salariés. } \\
\text { Source : Institut für Mittelstand, Bonn, 07/2004. }\end{array}$} \\
\hline
\end{tabular}


Un rôle socio-économique déterminant

Une industrie et des services compétitifs

Une moitié des PME est tributaire de la demande intérieure, ...

Le Mittelstand représente la colonne vertébrale de l'économie. Son poids a légèrement diminué ces dernières années, mais reste toujours très déterminant. S'il englobe $99 \%$ des entreprises assujetties à la TVA, son poids dans le CA total des entreprises allemandes s'élève à un peu plus de $41 \%(41,2 \%$ en 2002 contre 43,4\% en 2000). II emploie un peu plus de $70 \%$ des salariés et forme plus de $80 \%$ des apprentis. A lui seul, il réalise près de la moitié du PIB allemand. Sa contribution à l'investissement brut est à peine plus faible (46\% en 2000). Les entreprises sont souvent jeunes (un peu plus d'un tiers d'entre elles existent depuis moins de 10 ans) et assurent le renouvellement du tissu économique : on compte en moyenne quelque 500000 créations pour environ 420000 fermetures annuelles au cours des dernières années.

La situation économique et financière des PME reste fortement marquée par quatre années de stagnation, comme le révèle l'indicateur de la conjoncture du secteur, calculé par la Kreditanstalt für Wiederaufbau (KfW). En 2003, cet indicateur a atteint son niveau le plus bas depuis sa création en 1992. Mais après 4 ans de baisse, la situation s'améliore. Tandis que le BTP reste toujours à la traîne, le commerce, le secteur manufacturier et surtout les services ressentent la reprise et ont commencé l'année 2004 avec plus d'optimisme. Au plan de l'emploi, la situation d'ensemble reste néanmoins tendue depuis 2001. En 2003, la part des PME qui ont réduit leur effectif a dépassé de $13 \%$ celles qui ont embauché. Pour 2004, la prudence règne : seulement $12 \%$ envisageaient au début de l'année d'augmenter leurs effectifs. Par ailleurs, l'enquête de la KfW révèle que si le pourcentage des $\mathrm{PME}$ prêtes à investir à court terme est en hausse en 2004 (31\% contre $25 \%$ en 2003), il reste néanmoins en dessous de la moyenne à long terme (48\%).

C'est surtout l'atonie de la demande intérieure qui affecte le Mittelstand: la moitié des entreprises dépend presque exclusivement de la consommation allemande ; hors secteur manufacturier (et la branche de la machine-outil, leader mondial), cette proportion atteint trois quarts. Cette situation contraste nettement avec celle des grandes entreprises qui ont un taux moyen à l'exportation d'environ $30 \%$ et qui profitent pleinement de la reprise tirée par la demande extérieure. Le Mittelstand, quant à lui, arrive de moins en moins à remplir son rôle traditionnel de stabilisateur conjoncturel.

Mittelstand et exportation $\left.{ }^{\star}\right)$ (en \%)

\begin{tabular}{|lccc|}
\hline Industrie chimique & $\begin{array}{c}\text { Part du Mittelstand dans } \\
\text { l'exportation de la branche }\end{array}$ & $\begin{array}{c}\text { Taux } \\
\text { d'exportateurs }\end{array}$ & $\begin{array}{c}\text { Taux du CA } \\
\text { export / CA global }\end{array}$ \\
Production de produits plastiques & 7,8 & 51,5 & 25,9 \\
Industrie du papier & 34,9 & 49,5 & 21,1 \\
Machines outils & 18,9 & 44,1 & 21,5 \\
Voitures / pièces détachées & 25,4 & 42,4 & 32,0 \\
Meubles, bijoux, jeux, etc. & 1,4 & 35,3 & 21,3 \\
Produits métallurgiques & 45,7 & 22,5 & 18,0 \\
Commerce de détail & 46,0 & 20,5 & 19,1 \\
Energie & 36,8 & 12,9 & 6,8 \\
\end{tabular}

Source : Institut für Mittelstand, Unternehmensgrössenstatistik 2001/2002.*) Chiffres pour 1999. **) Pourcentage des entreprises exportatrices du Mittelstand par rapport à l'ensemble des entreprises de la branche. ${ }^{* *}$ ) Par branche pour les entreprises du Mittelstand.

... l'autre est le fief de hidden champions mondiaux
Cette dépendance de la demande intérieure doit cependant être nuancée. Dans certaines branches du Mittelstand, le taux d'exportateurs est proche de $50 \%$ (chimie, produits plastiques, papier, machines outil, métallurgie ou voitures). Ces branches profitent donc pleinement de la demande internationale, et leurs entreprises sont également les plus actives en matière d'emploi. Pour d'autres, cette ouverture est moins forte, comme pour les producteurs de meubles et bijoux, le commerce de détail ou le secteur de l'énergie. Ces branches dépendent 
donc davantage du marché intérieur allemand. Par ailleurs, presque toutes les branches du Mittelstand possèdent une caractéristique particulière : la présence de hidden champions, c'est-à-dire d'entreprises très spécialisées qui sont des leaders mondiaux dans leur domaine. En Allemagne, environ $20 \%$ des PME font partie de ce groupe - un record mondial. II comporte des Mittelständler de toutes catégories - de la TPE à la PME de 499 salariés, des entreprises de l'est, ainsi que des jeunes pousses.

Aujourd'hui, le Mittelstand est confronté à un environnement en forte mutation. Comme toutes les entreprises, les PME doivent s'adapter à la déréglementation et à la mondialisation. Mais le Mittelstand est également frappé par des problèmes plus spécifiques: il s'agit notamment de sa sous-capitalisation, du changement générationnel et des problèmes de financement, liés last but not least à Bâle II, le nouvel accord réglementaire pour les banques. Pour accompagner ces évolutions, le gouvernement de Berlin a commencé à assouplir le cadre réglementaire. La réforme de l'imposition des entreprises, entrée en vigueur début 2001, constitue une réelle avancée. Non seulement, elle réduit légèrement la charge fiscale pour l'ensemble des entreprises, mais elle met surtout fin à la longue pénalisation des Mittelständler par rapport aux sociétés de capitaux en permettant la défalcation de la taxe professionnelle dans l'imposition sur le revenu, une mesure réservée aux entreprises en nom personnel et sous loi civile, dont la charge fiscale est maintenant inférieure de plus de $3 \%$ à celle d'une société en capitaux dans la même situation. Quant au règlement concernant les licenciements, le gouvernement fédéral est revenu en 2004 à l'état de la réglementation de 1996, ramenant à 10 salariés le seuil à partir duquel s'applique la loi de protection contre le licenciement (le seuil avait été abaissé à 5 salariés en 1999). Les PME disposent ainsi d'un peu plus de flexibilité, mais cela ne suffit pas pour inciter à l'embauche, car la contribution du secteur à l'amélioration de la situation de l'emploi reste inférieure à son potentiel.

Le contexte conjoncturel, mais aussi le comportement plus sélectif des banques en matière de crédit et l'arrivée de Bâle II, sont en train de remodeler le bilan des PME. Le Mittelstand est traditionnellement pénalisé par son niveau trop faible en fonds propres. Avec un taux moyen des fonds propres rapporté au bilan de $20 \%$, les Mittelständler restent loin derrière la concurrence internationale (45 \% aux USA, $41 \%$ au Royaume-Uni, $34 \%$ en France, $22 \%$ au Japon). Le secteur est conscient du problème et a entrepris des efforts pour améliorer la situation. Mais il s'agit surtout des plus grandes PME et des sociétés de capitaux du secteur manufacturier: donc d'entreprises qui ont traditionnellement des taux de fonds propres plutôt élevés. Régionalement, cette tendance est plus marquée à l'ouest qu'à l'est, marqué structurellement par un manque de grandes PME. Certes, dans les bilans des entreprises du Mittelstand n'apparaît pas toujours l'ensemble des capitaux de garantie (haftende Eigenmittel), mais la situation reste insatisfaisante: le niveau en fonds propres du Mittelstand n'atteint même pas un tiers de celui des grandes entreprises.

Un autre problème concerne le vieillissement et l'inévitable succession à la tête des entreprises. En Europe, l'Allemagne est le pays le plus touché par le phénomène: plus de 70000 entreprises sont transmises chaque année, concernant plus de 900000 emplois (en France, un peu plus de 43000 entreprises seulement pour 160000 salariés). Dans la plupart des cas, la succession s'annonce difficile, faute de repreneur au sein de la famille. De plus en plus de PME sont donc obligées de faire appel à un successeur externe. Et ce problème concerne un nombre croissant d'entreprises, surtout des petites (ce qui relativise quelque peu le nombre de salariés concernés). L'institut de recherche sur le Mittelstand (IfM) estime que le nombre de fermetures faute de repreneur passera de 5000 en 2000 à 5900 en 2005. Le risque d'une perte de connaissances et d'infrastructures induit par l'ensemble de ces tendances, surtout au niveau local, est indéniable.
Commentaire [B1]: Oder : européen?

non, non, car les entreprises

américaines et japonaises sont moins Le c oriéntées à l'export (les PME bien sûr) évolue favorablement
Amélioration de la traditionnelle faiblesse en fonds propres
Le changement démographique au coeur des préoccupations

Commentaire [B2]: Comprends pas...

voir nouvelle verio 
Successions : un régime fisca dans la moyenne européenne

Un facteur essentiel dans tout projet de succession, surtout du point de vue des héritiers, est le traitement fiscal. Une comparaison européenne montre néanmoins que la situation n'est guère pénalisante pour les héritiers allemands : le montant de l'impôt s'élève en moyenne à $4 \%$ de la valeur de l'entreprise pour les descendants directs. L'Allemagne se place ainsi à un niveau moyen : en France (16\%), aux Pays-Bas (25\%) ou au Japon (30\%) les taux sont nettement plus élevés ; tandis qu'en Irlande, au Luxembourg et au Royaume-Uni, les petites entreprises sont léguées aux enfants sans impôts. Ce régime relativement favorable pourrait cependant évoluer. Actuellement, il est basé sur un traitement très avantageux des patrimoines d'entreprise, immobiliers et fonciers par rapport au reste du patrimoine (argent liquide et valeurs mobilières). Depuis 2002, le Tribunal constitutionnel fédéral examine une plainte en constitutionnalité déposée par la Cour fédérale des finances ; le verdict est attendu pour 2005.

La transmission des entreprises du Mittelstand ${ }^{*}$

\begin{tabular}{|lccc|}
\hline & Nombre d'entreprises & $\%$ & Nombre de salariés \\
Total & 70900 & $\mathbf{1 0 0}$ & $\mathbf{6 7 8} \mathbf{0 0 0}$ \\
Session à des membres de la famille**$)$ & 31000 & 43,8 & 351000 \\
Vente à des concurrents & 15000 & 21,1 & 114000 \\
Vente à des cadres extérieurs & 11700 & 16,5 & 107000 \\
Vente à des salariés $(M B O)$ & 7300 & 10,2 & 72500 \\
Fermeture sans successeur & 5900 & 8,3 & 33500 \\
\hline
\end{tabular}

Source : Institut für Mittelstand, Bonn (www.ifm-bonn.org). *) Prévisions 2005 pour les entreprises au CA supérieur à $\left.50000 €{ }^{* *}\right)$ Vente, legs, donation, etc.

Transmissions : peu de mesures fiscales spécifiques...

... mais soutien logistique et simplification des procédures

Bâle II : le financement du Mittelstand se réorganise
Le vieillissement des entrepreneurs est un problème européen. La Commission européenne estime qu'un tiers des entreprises de l'UE feront l'objet d'une transmission dans les dix prochaines années. Elle a recensé six domaines de mesures facilitant la transmission, dont des mesures fiscales (à destination des salariés et des tiers) et des aides matérielles et logistiques pour les entrepreneurs. Seule la Belgique a pris des mesures dans les six domaines; l'Allemagne est en deuxième position avec un retard dans la mise en place d'instruments appropriés pour financer des cas spécifiques de transmission. II y existe seulement un programme de financement pour les opérations de $\mathrm{MBO} / \mathrm{MBI}$ destiné aux capital-risqueurs pour leur permettre de refinancer leurs participations dans le cadre des cessions d'entreprises. D'autres mesures ne sont pas envisagées, par exemple dans le domaine des abattements fiscaux sur les droits de succession et de donation. Ceux-ci étant perçus par les Länder, il faudrait une initiative conjointe de ces derniers pour modifier la législation actuelle.

A l'avenir, des améliorations sont surtout à prévoir dans le domaine logistique et du conseil. La priorité sera donnée au perfectionnement des mesures de soutien actuel. Le portail «www.nexxt.org » en sera la clé de voûte. II est prévu de le transformer en un guichet unique pour toutes les questions de transmission d'entreprises, intégrant des bases de données sur les entreprises à transmettre (comme l'actuel site www.change-online.de), recensant les consultants spécialisés et offrant des séminaires et des formations. Le rôle de la $K f W$-Bankengruppe dans ces activités est central. II s'amplifiera encore à l'avenir, résultat de la récente fusion entre la KfW et la DtA (Deutsche Ausgleichsbank) dont est issue la KfW-Bankengruppe, conformément à la loi Förderbankenneustrukturierungsgesetz de l'été 2003. Par ailleurs, il est prévu de renforcer le lien recherche-entreprise en incitant les universités à intégrer les questions de transmission dans leurs programmes d'études et de recherche.

Préparer en bon ordre les questions concernant la succession intéressera de plus en plus les banques, confrontées à un environnement réglementaire en pleine mutation à cause du nouvel accord de Bâle sur les fonds propres. II implique le passage d'un système traitant chaque risque de manière identique (Bâle I avec sa norme homogène de $8 \%$ de fonds propres qui s'est soldée par 
la subvention des mauvais risques par les bons risques) à une individualisation des risques. Chaque banque devra à l'avenir adapter les conditions de crédit au risque et à la probabilité de défaut de l'entreprise, mesurés par un rating soit externe (agences spécialisées) soit interne. Après ratification par la Commission européenne et transformation en loi nationale, Bâle II entrera progressivement en vigueur à partir du 31 décembre 2006.

En Allemagne, les propositions du comité de Bâle, exposées pour la première fois en 1999, ont reçu un écho particulièrement fort, surtout chez les PME qui risquent plus que les grandes entreprises de subir une dégradation de leurs conditions de financement: chez les premières, la part des crédits bancaires s'élève à $45 \%$ du bilan, mais à seulement $9 \%$ chez les secondes. La dépendance du crédit bancaire est telle que celui-ci joue partiellement le rôle de quasi-fonds propres, une conséquence de la relation très étroite entre le patron et son banquier (principe de la Hausbank).

A la suite d'une série de papiers consultatifs et d'études d'impact réalisée entre 1999 et 2003, accompagnés d'un lobbying intense de la part du Mittelstand, le règlement initial a connu une adaptation substantielle. L'accord final, présenté le 26 juin 2004, contient trois mesures favorables au Mittelstand, en réponse à ses critiques:

- les crédits aux entreprises dont le volume total de crédits bancaires ne dépasse pas 1 million $€$ peuvent être traités par la banque comme des crédits aux particuliers, exigeant ainsi moins de réserves en fonds propres ;

- concernant les crédits aux entreprises au CA inférieur ou égal à 50 millions $€$ et au volume de crédit supérieur à 1 million $€$ (auprès de l'établissement bancaire concerné), le calcul du risque de défaut dépendra de la taille de l'entreprise : plus l'entreprise sera petite, plus la probabilité de défaut sera réduite ;

- dans le cas des crédits à long terme, Bâle II exige des fonds propres supplémentaires (sauf si l'entreprise concernée relève de la catégorie 1) ; cependant, l'autorite de contrôle nationale a le choix d'exonérer de ce supplément des entreprises avec un CA annuel et un bilan de moins de 500 millions $€$. L'autorité allemande, la Bundesamt für Finanzdienstleistungsaufsicht (BaFin), a annoncé son intention de pratiquer cette exonération.

Ces améliorations offriront à la plupart des PME des conditions de financement proches, voire identiques à celles d'aujourd'hui. Par rapport à la version initiale, l'ancien niveau d'exigence de fonds propres de $8 \%$ ne sera atteint qu'à partir d'un rating $\mathrm{BB}$ (BB+ auparavant). Reste un litige sur la pratique du rating. Basée sur les chiffres de bilan, elle traite toutes les entreprises de la même manière et ne peut guère prendre en compte les particularités des PME (notamment le fait que le bilan ne contient pas tous les éléments, surtout l'immatériel de l'entreprise). Ensuite, les résultats des bilans des entreprises sont très corrélés - d'où le reproche fait à Bâle II d'une procyclicité : une dégradation des bilans des entreprises inciterait ainsi les banques à durcir leur offre de crédit (avec un risque d'un resserrement global des conditions de crédit, ce qui dégraderait encore davantage la situation des entreprises. II est certain que le nouveau règlement se traduira par une plus grande disparité des conditions. Pour les entreprises représentant de mauvais risques et/ou se montrant trop rigides au plan de la transparence, le financement sera plus cher. Cette exigence d'une plus grande transparence est un aspect auquel le Mittelständler, mais également le banquier, devront encore s'accoutumer, habitués par le principe de la banque-maison à une relation privilégiée, souvent basée sur la confiance.

En déduire un affaiblissement du principe de la Hausbank serait néanmoins exagéré. Les banques resteront le partenaire privilégié du Mittelstand (à $90 \%$ des caisses d'épargne, des Landesbanken et des banques mutualistes) pour les deux volets du Hausbankprinzip: les questions de financement et l'accès au aides offertes par les 23 promoteurs publics, dont surtout la KfW-Bankengruppe et les Landesanstalten für Aufbaufinanzierung (LfA) des Länder. A l'avenir, les banques auront un rôle plus important de conseil à jouer, car les entreprises
Crédit bancaire : $45 \%$ du bilan des PME

Accord Bâle II : 3 mesures favorables au Mittelstand

Le rating et la procyclicité restent un souci

La relation banque-entreprise restera déterminante 
True-Sales-Initiative : amélioration de la rentabilité des banques

Une politique de soutien pas encore optimale

pour lesquelles le financement sera plus difficile avec Bâle II seront incitées à recourir à d'autres formes de financement, comme le leasing, le factoring (vente de créances à un tiers), les obligations d'entreprises et, dans le cas d'une société anonyme, l'émission d'actions. Si ces instruments sont encore réservés à une minorité de PME, la situation évolue rapidement, comme le montre l'émission de la première obligation perpétuelle d'une entreprise du Mittelstand; il s'agit de l'entreprise Claas, producteur de machines agricoles.

Du côté des banques, les instruments se modernisent également. La Truesales-Initiative des banques et de la $\mathrm{KfW}$, qui permet de restructurer les portefeuilles de crédit par la vente sur le marché (les crédits sont cumulés et transformés dans un titre négociable permettant à la banque de récolter des liquidités immédiates), est un premier pas important dans cette direction. Elle renforce les banques dans leurs efforts pour assainir leur bilan et améliorer leur rentabilité, condition nécessaire d'une consolidation ultérieure des trois piliers du secteur bancaire allemand (privé, public, mutualiste).

La politique des banques de soutien est également en train de changer. Depuis la loi Förderbankenneustrukturierungsgesetz de 2003, elles doivent - en échange de la préservation de la garantie de l'Etat - davantage veiller à ne pas pratiquer de distorsion de la concurrence. Cela incite les banques commerciales à proposer encore un peu plus leurs propres solutions financières au détriment des moyens offerts par les promoteurs publics. Pourtant, les aides financières destinées au Mittelstand ont progressé continuellement ces dernières années : en 2002, plus d'un tiers des moyens de soutien aux entreprises est allé à aux PME (700 millions $€$ sur un total de 1,8 milliard $€$ ). Le problème est que ces dernières ne disposent pas toujours des informations nécessaires quant à l'existence des fonds; en outre, les volumes exigés (montant minimum pour une promotion) sont souvent trop élevés. D'autres instruments, comme surtout les aides indirectes (garanties, cautionnements), sont de plus en plus utilisés.

MALGRE LeS Difficultes auxquels il doit faire face, le Mittelstand représente toujours le gisement essentiel de dynamisme et d'innovation pour l'Allemagne. Mais il subit encore de plein fouet les rigidités structurelles qui inhibent la croissance outre-Rhin : moins internationalisé que les très grandes entreprises, les PME sont bien plus qu'elles tributaires de la réglementation nationale. Elles disposent certes des atouts nécessaires pour s'ajuster aux réorganisations en cours, comme Bâle II, le problème des successions et les mutations de la relation banque-entreprise. Mais pour que le Mittelstand déploie vraiment son potentiel, il faudra à l'avenir plus que des mesures concernant la promotion ou le cadre réglementaire du secteur, mais un nouvel équilibre entre l'économie et la société, laissant plus de place à l'initiative individuelle et à la prise de risque. Le gouvernement fédéral a commencé de s'engager dans cette voie avec ses réformes sociales. Mais beaucoup reste encore à faire, surtout au plan des prélèvements sociaux et de la flexibilisation du marché du travail. L'enjeu est crucial : le Mittelstand constitue la base même du tissu économique et social allemand.

\section{Indications bibliographiques}

Bundesverband deutscher Banken, « Mittelstandfinanzierung vor neuen Herausforderungen », Série Daten, Fakten, Argumente, janvier 2003.

Commission européenne, Transmission d'entreprises - La continuité grâce à un nouveau départ, Bruxelles, août 2003.

DEUTSCHE BUNDESBANK, « Zur wirtschaftlichen Situation kleiner und mittelständischer Unternehmen in Deutschland », Monatsbericht, octobre 2003, et Wege aus der Krise - Wirtschaftspolitische Denkanstöße für Deutschland, mars 2003.

Hommel U., SchNeIDER H., «Financing the German Mittelstand », ElB-Papers, vol. 8, $\mathrm{n}^{\circ} 2,2003$.

Mittelstandsmonitor 2004, KfW-Bankengruppe, 2004.

Traistra G., « Basel II - Aktueller Stand und Auswirkungen auf die Mittelstandfinanzierung », KfW, 2003. 